\title{
ARTICLE \\ Platelet activating factor receptor regulates colitis-induced pulmonary inflammation through the NLRP3 inflammasome
}

\author{
Gang Liu ${ }^{1,2,3}$, Sean W. Mateer ${ }^{1,2,3}$, Alan Hsu ${ }^{2,4}$, Bridie J. Goggins ${ }^{1,2,3}$, Hock Tay ${ }^{1,2}$, Andrea Mathe ${ }^{1,2,3}$, Kening Fan ${ }^{1,2,3}$, Rachel Neal $^{1,2,3}$, \\ Jessica Bruce ${ }^{1,2,3}$, Grace Burns ${ }^{1,2,3}$, Kyra Minahan ${ }^{1,2,3}$, Steven Maltby ${ }^{2,5}$, Michael Fricker ${ }^{2,4}$, Paul S. Foster ${ }^{1,2}$, Peter A. B. Wark ${ }^{2,4}$, \\ Philip M. Hansbro ${ }^{1,2}$ and Simon Keely ${ }^{1,2,3}$
}

Extra-intestinal manifestations (EIM) are common in inflammatory bowel disease (IBD). One such EIM is sub-clinical pulmonary inflammation, which occurs in up to $50 \%$ of IBD patients. In animal models of colitis, pulmonary inflammation is driven by neutrophilic infiltrations, primarily in response to the systemic bacteraemia and increased bacterial load in the lungs. Platelet activating factor receptor (PAFR) plays a critical role in regulating pulmonary responses to infection in conditions, such as chronic obstructive pulmonary disease and asthma. We investigated the role of PAFR in pulmonary EIMs of IBD, using dextran sulfate sodium (DSS) and anti-CD40 murine models of colitis. Both models induced neutrophilic inflammation, with increased TNF and IL$1 \beta$ levels, bacterial load and PAFR protein expression in mouse lungs. Antagonism of PAFR decreased lung neutrophilia, TNF, and IL$1 \beta$ in an NLRP3 inflammasome-dependent manner. Lipopolysaccharide from phosphorylcholine (ChoP)-positive bacteria induced NLRP3 and caspase-1 proteins in human alveolar epithelial cells, however antagonism of PAFR prevented NLRP3 activation by ChoP. Amoxicillin reduced bacterial populations in the lungs and reduced NLRP3 inflammasome protein levels, but did not reduce PAFR. These data suggest a role for PAFR in microbial pattern recognition and NLRP3 inflammasome signaling in the lung.

Mucosal Immunology (2019) 12:862-873; https://doi.org/10.1038/s41385-019-0163-3

\section{INTRODUCTION}

Inflammatory bowel disease (IBD) including Crohn's diseases (CD) and ulcerative colitis (UC) are chronic diseases of gastrointestinal (GI) tract. ${ }^{1}$ Although the pathology between CD and UC differs, they share a number of common characteristics and comorbidities, the chief of which is arguably the loss of epithelial barrier function. ${ }^{2}$ This loss of epithelial integrity promotes bacteraemia in IBD patients ${ }^{3-5}$ and these pathologies are apparent in animal models of colitis., ${ }^{6,7}$ In IBD patients, bacteraemia can promote extra-intestinal inflammation and approximately half of IBD patients have secondary organ pathologies that contribute significantly to disease morbidity. ${ }^{8}$ An increasing number of studies suggest that abnormal lung pathology is a common feature of $\mathrm{IBD}_{1}^{9,10}$ with subclinical disease observed in over one third of IBD patients. ${ }^{11}$ While some therapies can promote lung damage, ${ }^{12}$ in the majority of cases pulmonary disease associated with IBD is idiopathic, and animal studies have demonstrated that lung pathologies similar to those observed in IBD patients are evident in animal models of colitis. ${ }^{7,13,14}$ Our recent studies suggest that pulmonary manifestations of colitis arise from neutrophilia associated with loss of intestinal epithelial integrity and subsequent bacteraemia. ${ }^{7}$ An increased bacterial load in the lungs may be the initiating factor for the observed pulmonary neutrophilia, although the pathway initiating this response is unclear.

In respiratory diseases, such as chronic obstructive pulmonary disease (COPD) and asthma, the majority of acute exacerbations are driven by bacterial and viral infection, ${ }^{15}$ with pathogens often exploiting cellular receptors and adhesion molecules upregulated during inflammatory processes. ${ }^{16}$ One such receptor, plateletactivating factor (PAFR) is a G protein-coupled receptor expressed on epithelial and endothelial cells, macrophages and neutrophils. $^{17}$ PAFR is a hypoxia-responsive gene ${ }^{18}$ and PAFR protein levels are increased in inflamed lungs from patients with asthma ${ }^{19}$ and COPD. ${ }^{20}$ PAFR has affinity for bacterial phosphorylcholine (ChoP) moieties ${ }^{21}$ and increased expression of PAFR is associated with both Gram-negative and Gram-positive infection. ${ }^{22}$ Thus, in conditions of increased pulmonary stress of infection, PAFR may represent a crucial mediator of inflammation and inhibition or antagonism of PAFR has been considered as a therapeutic strategy for the management of pulmonary disease. ${ }^{23,24}$

In the pulmonary inflammation associated with colitis, bacteraemia is an initiating factor and pulmonary inflammation is dependent on neutrophil recruitment from the bone marrow. ${ }^{7}$ While these animals exhibit increased bacterial load in the lungs, the mechanism initiating neutrophil recruitment to the lung is unclear. Here, we hypothesized that increased bacterial burden in the lung, driven by colitis-associated bacteremia, activates antimicrobial responses that drive neutrophil recruitment. Using dextran sulfate sodium (DSS) and anti-CD40 models of colitis, we identified increases in PAFR in pulmonary epithelial cells and neutrophils associated with increased bacterial load and NLRP3 inflammasome activation in the lungs. Antagonism of PAFR

\footnotetext{
${ }^{1}$ School of Biomedical Sciences and Pharmacy, University of Newcastle, Callaghan, NSW, Australia; ${ }^{2}$ Hunter Medical Research Institute, New Lambton Heights, NSW, Australia;

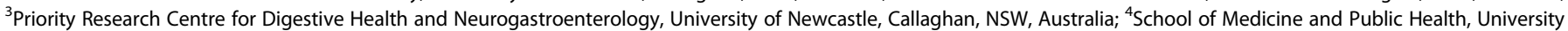
of Newcastle, Callaghan, NSW, Australia and ${ }^{5}$ School of Nursing and Midwifery, University of Newcastle, Callaghan, NSW, Australia Correspondence: Simon Keely (Simon.Keely@newcastle.edu.au)
} 
reduced neutrophil but not macrophage recruitment to the lung and reduced neutrophil extracellular traps (NETs) formation in the parenchyma. In vitro studies with pulmonary epithelial cells demonstrated NLRP3 activation, cleavage of caspase- 1 and IL-1 $\beta$ secretion in response ChoP-positive, but not ChoP negative lipopolysaccharide (LPS). However, this inflammasome activation was inhibited by PAFR antagonism. Further, PAFR induction and inflammasome activation in the lungs of DSS colitis animals was inhibited by antibiotic treatment. These data implicate PAFRmicrobe interactions as a key modulator of pulmonary inflammation in colitis and bacteremia, and suggest that PAFR is a microbial sensor regulating local inflammasome responses.

\section{RESULTS}

Experimental models of colitis induce lung inflammation

To explore colitis associated with lung inflammation, we employed an acute model of DSS-induced colitis as described previously. ${ }^{7,13}$ Colitis features were determined by measuring body weight and by endoscopy. DSS challenge resulted in significant weight loss by day 4 and further decrease over days 5-7 (Fig. 1a). Endoscopic assessment showed increasing mucosal inflammation and ulceration over time (Fig. 1b). DSS challenge increased total leukocytes, predominately macrophages and neutrophils, in bronchoalveolar lavage fluid (BALF) from mouse lungs (Fig. 1c). Histological analysis demonstrated that inflammatory cells were predominantly localized around the parenchyma and blood vessels in mouse lungs after 7 days of DSS challenge (Fig. 1d, e).

We next examined whether innate or adaptive responses were key to neutrophil recruitment to the lungs. To this end, we employed the anti-CD40 model to induce colitis in recombinase activating gene 1 (RAG1) deficiency (-/-) mice as they lack mature $\mathrm{T}$ and $\mathrm{B}$ cells. ${ }^{25}$ Significant weight loss was observed in the antiCD40-treated animals, peaking 3 days after disease induction (Fig. 1f). Endoscopic disease was observed within 2 days of induction and progressed over the course of the model (Fig. 1g). The anti-CD40 challenge also increased macrophages and neutrophil numbers in BALF and induced lung inflammation (Fig. $1 \mathrm{~h}-\mathrm{j}$ ) which were not observed in control mice that received isotype IgG control antibody.

Colitis increases PAFR proteins and activates NLRP3 involved inflammasome in mouse lungs

Our previous studies suggested microbial involvement in colitisinduced lung inflammation. ${ }^{7}$ In this study, we confirmed that DSSinduced colitis resulted in an increase of bacterial $16 \mathrm{~S}$ expression in mouse lungs compared to controls (Fig. 2a). Given the importance of PAFR in pulmonary infection during inflammatory disease, we measured PAFR proteins in mouse lungs in our model of DSS-induced lung inflammation and found significantly increased PAFR protein levels on day 7 post DSS (Fig. 2b). These data were mirrored in the anti-CD40 model with $16 \mathrm{~S}$ expression and PAFR protein significantly increased in mouse lungs by 9 days post challenge (Fig. 2c, d), indicating these are common features in colitis-induced lung inflammation.

To understand the role of PAFR in colitis-induced lung inflammation, we investigated cellular sources of PAFR in lungs. Given the consistent increase in pulmonary neutrophils observed in colitis models, ${ }^{7}$ lung sections were stained with PAFR and Ly6G by immunofluorescence. Increased PAFR staining was observed in the parenchymal area, and alveolar epithelial cells (according to morphology) and neutrophils were the predominant cell types to produce PAFR in mouse lungs (Fig. 2e). To confirm the cellular source of PAFR, we isolated single cell suspensions from mouse lungs and co-stained cell phenotyping markers and PAFR to identify and enumerate by flow cytometry. PAFR ${ }^{+}$epithelial cells (CD45 ${ }^{-}$Epcam $^{+}$, Fig. $2 f$ and Supplementary Fig. S1a), macrophages $\left(\mathrm{CD} 45^{+} \mathrm{F} 4 / 80^{+}\right.$, Fig. $2 \mathrm{~g}$ and Supplementary Fig. S1b) and neutrophils $\left(\mathrm{CD} 45^{+} \mathrm{CD} 11 \mathrm{~b}^{+} \mathrm{Ly}_{6 \mathrm{G}}{ }^{+}\right.$, Fig. $2 \mathrm{~h}$ and Supplementary Fig. S1c) were significantly increased in mouse lungs after 7 days DSS challenge. There were no changes to $\mathrm{PAFR}^{+}$dendritic cell $\left(\mathrm{CD} 45^{+} \mathrm{CD} 11 \mathrm{c}^{+}\right)$or eosinophil $\left(\mathrm{CD} 45^{+} \mathrm{CD} 11 \mathrm{c}^{-} \mathrm{SigF} \mathrm{F}^{+}\right)$populations (Supplementary Fig. S2) in mouse lungs after 7 days DSS challenge when compared to controls.

In line with previous results, ${ }^{7}$ cytokine analysis showed significantly increased levels of TNF and IL-1 $\beta$ proteins in mouse lungs after DSS challenge (Fig. $3 a, b$ ). Given that $I L-1 \beta$ is downstream of inflammasome activation, we measured protein levels of molecules involved in inflammsome response in mouse lungs. DSS challenge led to significant increases in NLRP3 and mature caspase- 1 proteins in mouse lungs when compared to healthy controls, however pro-caspase- 1 proteins were not changed (Fig. $3 c$, d). To confirm that PAFR enhances IL-1 $\beta$ secretion by lung epithelial cells via inflammasome activation, we isolated PAFR ${ }^{+}$epithelial cells from mouse lungs after 7 days DSS challenge and controls. The protein levels of the molecules involved in NLRP3 signaling pathways were measured in cell lysates. There were significantly increased NLRP3, pro- and mature caspase-1 protein levels in epithelial cells from mouse lungs after 7 days DSS challenge compared to controls (Fig. 3e, f). Lung epithelial cells from DSS animals also had increased IL-1 $\beta$ compared to healthy controls (Fig. $3 \mathrm{~g}$ ).

Inhibition of PAFR reduces mouse lung inflammation after DSS challenge

To further investigate the role of PAFR in colitis-induced lung inflammation, we administered the PAFR antagonist, CV620926 to mice, either intranasally or intravenously from day 4 to 6 of DSS challenge. We chose these time-points based on onset of disease as measured by weight loss during DSS challenge. On day 7, DSS challenge increased total leukocytes, predominately macrophages and neutrophils, in the BALF, compared to control animals (Fig. 4a-c). CV6209 treatments with both intranasal and intravenous deliveries significantly reduced airway neutrophils, but not macrophages $(P=$ 0.1302 , DSS + CV6209 i.n vs DSS + PBS i.n; $P=0.0528$, DSS + CV6209 i.v vs DSS + PBS i.v) in the DSS model. Overall, increases in inflammatory cells in mouse lungs due to colitis were reduced with CV6209 treatment by both delivery approaches (Fig. 4d, e). Inhibition of PAFR by intravenous delivery of CV6209 also reduced DSSinduced TNF (Fig. 4f), IL-1 $\beta$ (Fig. 4g), and IL-6 protein (Fig. 4h) in mouse lungs compared to controls. With intranasal delivery of CV6209, only IL-1 $\beta$ was significantly reduced suggesting the lung is not the primary source of TNF and IL- 6 in these models.

PAFR regulates neutrophil recruitment in mouse lungs after DSS challenge

Given that antagonism of PAFR reduced neutrophils in BALF of DSS animals, we next examined lung tissue neutrophils and macrophages using flow cytometry. DSS challenge significantly increased neutrophils and macrophages in mouse lungs by 7 days post challenge (Fig. 5a-c and Supplementary Fig. S3). Antagonism of PAFR by CV6209 with both intranasal and intravenous approaches reduced neutrophils, but not macrophages in mouse lungs, mirroring our finding of these cells in BALF.

NETs play a critical role in neutrophil antimicrobial activity, ${ }^{27}$ and we next assessed whether antagonism of PAFR influenced NETs in lung tissue. The lung sections were assessed by immunofluorescence for expression of NET markers, myeloperoxidase (MPO), a peroxidase enzyme that is released by neutrophils, ${ }^{27}$ and histone $\mathrm{H}^{28}$ (Fig. 5d). DSS challenge increased the number of MPO and histone $\mathrm{H} 1$ positive cells in mouse lungs, however antagonism of PAFR significantly decreased these cells (Fig. 5e).

PAFR activates the NLRP3 inflammasome signaling pathway We have shown that antagonism of PAFR reduced IL- $1 \beta$ protein levels in mouse lungs after DSS challenge. Given that we observed 


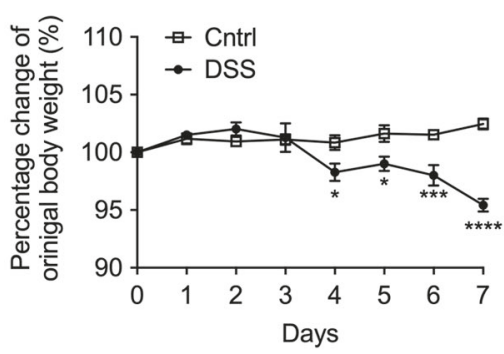

b

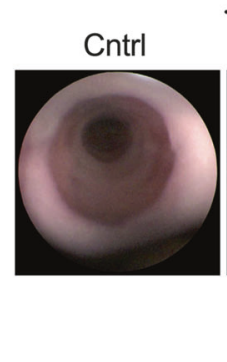

DSS

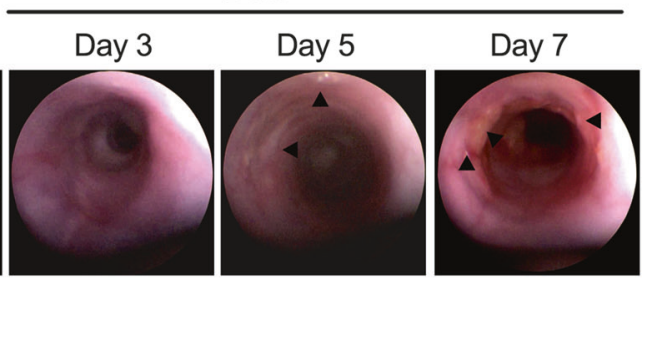

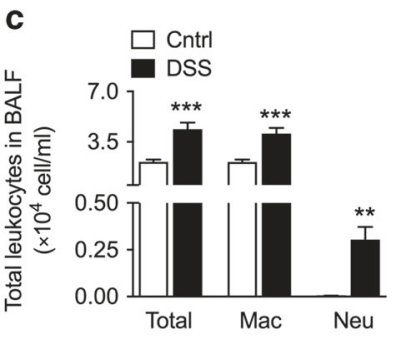

f

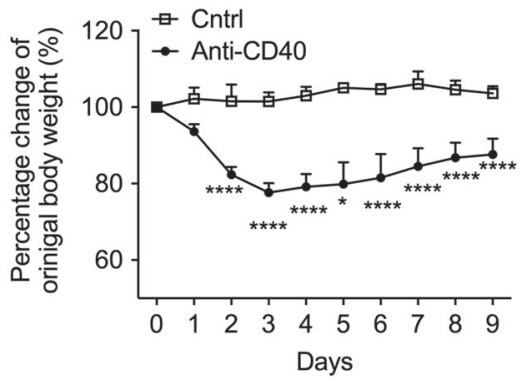

d

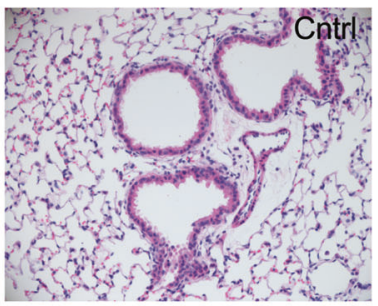

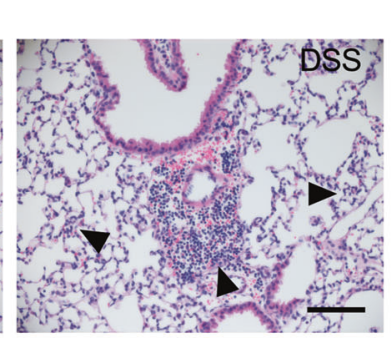

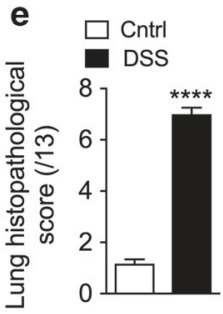

g

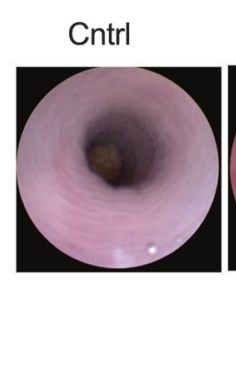

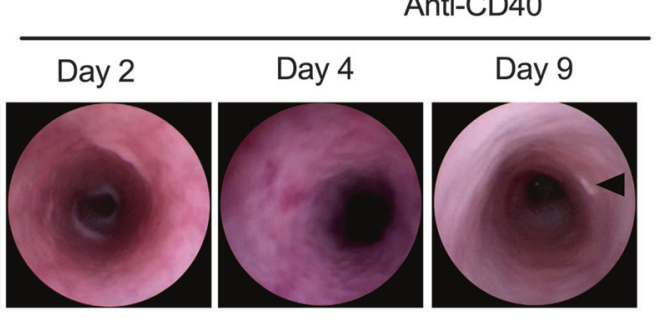
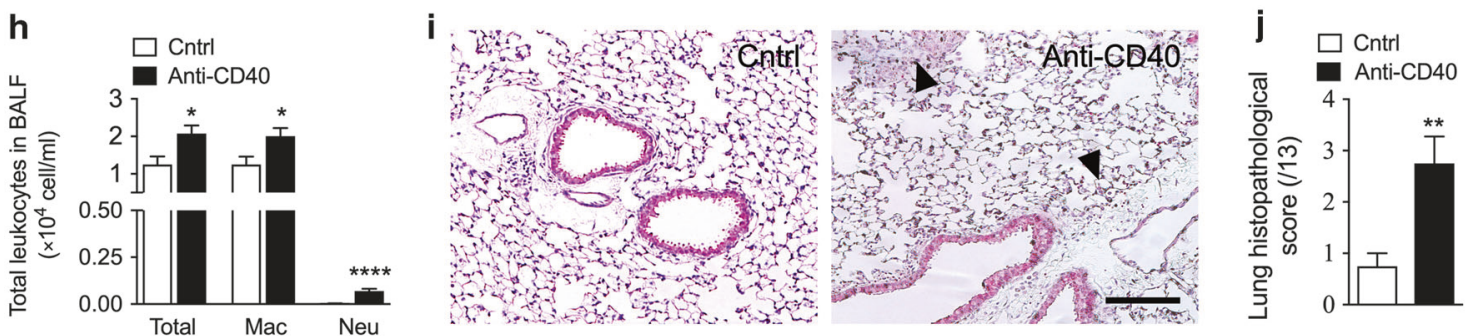

Fig. 1 Experimental models of colitis induces lung inflammation in mice. Mice received DSS in drinking water over 7 days. Control mice received plain water. Mouse body weight (a) and colonic endoscopy (b, arrows indicate lesions) were assessed during time-course of DSS challenge. c Total leukocytes and cell differential counts, including macrophages and neutrophils were enumerated in bronchoalveolar fluid (BALF). d Mouse lungs were collected, and lung sections were stained with H\&E, arrows indicate inflammation. e Mouse lung inflammation scores were assessed after 7 days DSS challenge. Mice were injected with monoclonal anti-CD40 antibody for 9 days. Control mice received IgG antibody. Mouse body weight (f) and colonic endoscopy (g, arrows indicate lesions) were assessed during time-course of anti-CD40 antibody challenge. $\mathbf{h}$ Total leukocytes and cell differential counts, including macrophages and neutrophils were enumerated in BALF. $\mathbf{i}$ Mouse sections were stained with $\mathrm{H} \& \mathrm{E}$, arrows indicate inflammation. $\mathbf{j}$ Mouse lung inflammation scores were assessed after 9 days anti-CD40 antibody challenge. $n=6-8$, mean \pm SEM. Statistical analysis was assessed by two-way ANOVA (a, f) and Student's $t$-test (c, e, $\mathbf{h}, \mathbf{j})$, * $P<0.05$, ${ }^{* *} P<0.01,{ }^{* * *} P<0.001,{ }^{* * * *} P<0.0001$ compared to control mice

inflammasome activation in the lungs of DSS-induced colitis animals, we next examined whether PAFR antagonism reduced inflammasome signaling in mouse lungs after DSS challenge and found that intranasal administration of CV6209 reduced both NLRP3 and mature caspase-1 proteins in mouse lungs (Fig. 6a, b).

Because alveolar epithelial cells were a source of PAFR in the lung tissue of DSS animals and microbial ChoP is a ligand for $\mathrm{PAFR}^{21}$ we examined whether ChoP was sufficient to drive PAFR expression. Human alveolar epithelial cells (A549) were challenged by LPS isolated either from ChoP positive and ChoP negative bacteria, and PAFR proteins were measured in cell lysate by immunoblot. LPS stimulation from ChoP positive bacteria significantly increased PAFR proteins in epithelial cells, whereas PAFR protein levels did not change after LPS challenge from ChoP negative bacteria (Fig. 6c). We next investigated whether PAFR interaction with ChoP directly regulated NLRP3 inflammasome activation in A549 cells. Challenge with ChoP-positive LPS significantly increased NLRP3, pro-caspase and mature caspase protein expression, however treatment with CV6209 $1 \mathrm{~h}$ prior to ChoP challenge, significantly reduced ChoP-driven expression of 


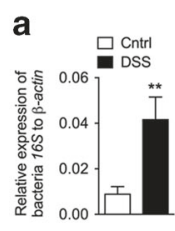

b

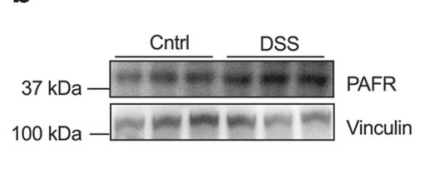

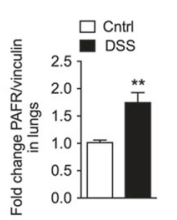

C

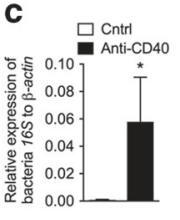

d

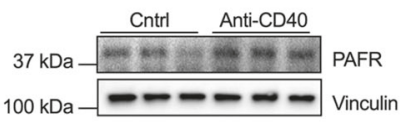

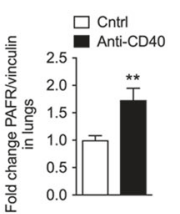
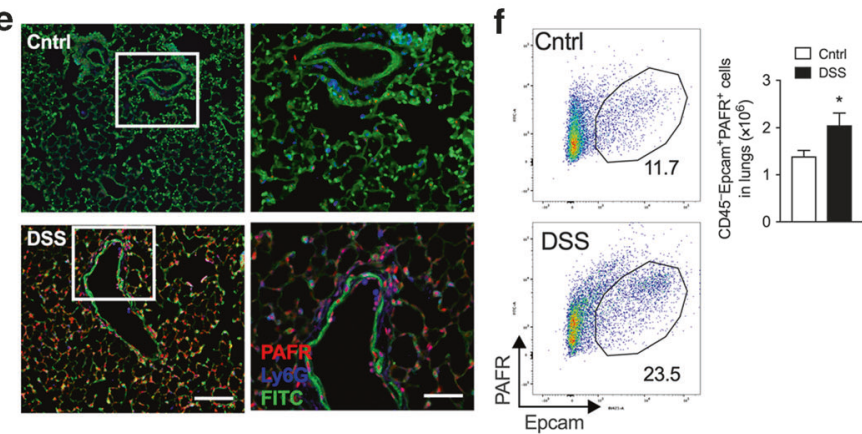

g
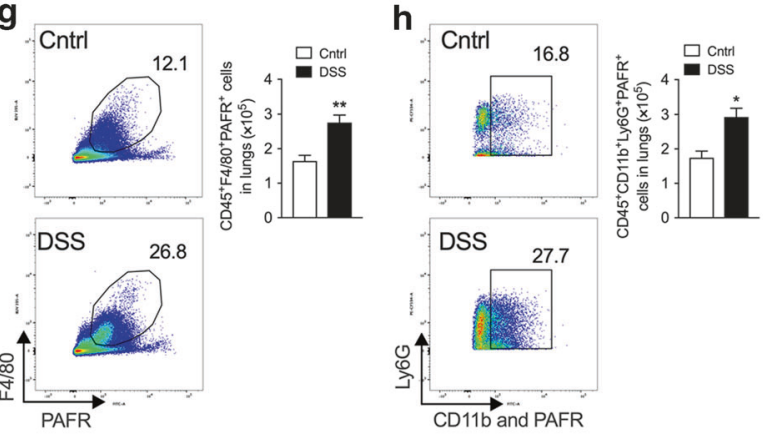

Fig. 2 PAFR expression and cellular sources of PAFR in mouse lungs. a Bacterial 165 expression was measured in mouse lungs by qPCR after 7 days DSS challenge. b PAFR protein in mouse lungs was measured after 7 days DSS challenge by immunoblot (left), and fold change of densitometry of PAFR normalized to vinculin (right). c Bacterial 165 expression was measured in mouse lungs by qPCR after 9 days anti-CD40 antibody challenge. d PAFR protein in mouse colons was measured after 9 days anti-CD40 antibody challenge by immunoblot (left), and fold change of densitometry of PAFR normalized to vinculin (right). e Lung sections from DSS or control animals were stained with PAFR (red) and ly6G (blue) by immunofluorescence. Auto-fluorescence in FITC shows cell morphology; scale bar: $200 \mu \mathrm{m}$. Single cells were obtained from mouse lungs, and CD45 Epcam ${ }^{+} \mathrm{PAFR}^{+}$(epithelial cells, f), CD45 ${ }^{+} \mathrm{F} 4 / 80^{+} \mathrm{PAFR}^{+}$(macrophages, g) and $\mathrm{CD} 45^{+} \mathrm{CD} 11 \mathrm{~b}^{+} \mathrm{Ly}^{-} \mathrm{GG}^{+} \mathrm{PAFR}{ }^{+}$cells (neutrophils, h) were enumerated by flow cytometry. $n=6-8$, mean \pm SEM. Statistical analysis was assessed by Student's $t$-test. ${ }^{*} P<0.05,{ }^{* *} P<$ 0.01 compared to control mice

all 3 proteins (Fig. $6 \mathrm{~d}$, e). LPS from ChoP negative bacteria did not increase NLRP3 inflammasome protein levels. Antagonism of PAFR also significantly reduced secreted IL-1 $\beta$ proteins in cell supernatant after challenge with ChoP positive LPS (Fig. 6f).

Antibiotic treatment reduces ChoP positive bacteria in lungs and regulates NLRP3 involved inflammasome in colitis-induced lung inflammation

Given the evidence that ChoP-PAFR interactions activated the NLRP3-mediated inflammasome in the lungs of DSS-treated animals, we investigated whether reducing microbial load in the lungs through antibiotic treatment would affect inflammasome activation during colitis. To this end, we treated mice with amoxicillin, either intranasally or intragastrically, during DSS challenge. Intranasal amoxicillin treatment did not influence DSS disease course as measured by body weight, however DSS animals receiving intragastric delivery of amoxicillin had significantly higher weight loss compared to DSS animals receiving vehicle (Supplementary Fig. S4a, b). Both intranasal and intragastric deliveries of amoxicillin led to significantly decreased total bacterial 165 expression in mouse lungs following DSS challenge, and 165 expression for ChoP positive bacteria, such as Klebsillia. pneumoniae and Pseudomonas. aeruginosa was also reduced in mouse lungs (Fig. 7a, b).

To investigate whether this reduced bacterial load influenced PAFR expression in colitis-induced lung inflammation, we measured PAFR and NLRP3-involved inflammasome proteins in mouse lungs after DSS challenge with and without amoxicillin treatment. Interestingly, intranasal antibiotic treatment did not change DSS-induced PAFR proteins levels in mouse lungs (Fig. 7c), however, NLRP3 and mature caspase-1 proteins were decreased in these animals when compared to DSS challenge with intranasal PBS treatment. Pro-caspase-1 proteins were not altered after DSS challenge. Similarly, intragastric amoxicillin treatment had no effect on the levels of PAFR in mouse lungs (Fig. 7d) but led to significantly decreased NLRP3 and mature caspase-1 proteins.

Histological scoring of lung inflammation in DSS animals with and without amoxicillin treatment showed that antibiotics reduced inflammation and neutrophil recruitment in mouse lungs (Supplementary Fig. S4c-d). Both intranasal and intragastric amoxicillin treatment decreased DSS-induced TNF, IL-1 $\beta$, and IL-6 protein in mouse lungs (Supplementary Fig. S5). These data suggest that bacterial burden does not regulate PAFR expression in itself, but is required for downstream PAFR inflammasome activation.

\section{DISCUSSION}

Secondary organ pathologies are common in IBD and there is increasing interest in the mucosal cross-talk between the gut and lung in inflammatory diseases. ${ }^{7,9}$ Our previous work has demonstrated that systemic inflammation is a driving factor in lung inflammation common to animal models of colitis. ${ }^{22}$ In this study, we identify expression of PAFR and PAFR-mediated inflammasome activation as regulators of neutrophil recruitment to the lung when microbial-burden is increased. In two distinct models of colitis, increased lung PAFR expression was associated with increased airway inflammation, neutrophils in the BALF and increased bacterial burden in the lungs. PAFR expression was localized to epithelial cells and neutrophils and associated with NET-formation in the lung tissue of DSS colitis animals. Immunoblot analysis identified increased NLRP3 inflammasome activation in DSS animals leading to IL-1 $\beta$ secretion in lung tissues and these responses were inhibited by antagonism of PAFR. Mechanistic studies indicated that PAFR mediates NLRP3 activation and caspase- 1 cleavage in response to interactions between microbial ChoP and PAFR, ${ }^{21}$ and that reduction of microbial load with antibiotics is sufficient to reduced inflammasome activation in the lungs of DSS animals, but not PAFR expression. These data suggest that PAFR acts as a pattern recognition receptor (PRR) for ChoP and is a mucosal mediator of the NLRP3 inflammasome.

PAFR is a hypoxia-responsive $G$ protein-coupled receptor ${ }^{18}$ on multiple cell types in lungs, and the increased expression of PAFR has been observed in many inflammatory-driven airway diseases, particularly those with an infectious component. ${ }^{29}$ PAFR protein is increased in both large and small airway epithelium and alveolar 
a

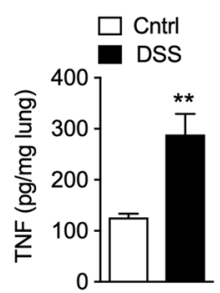

b

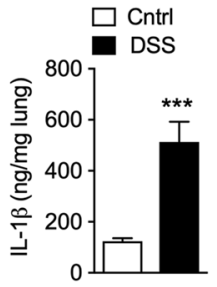

e

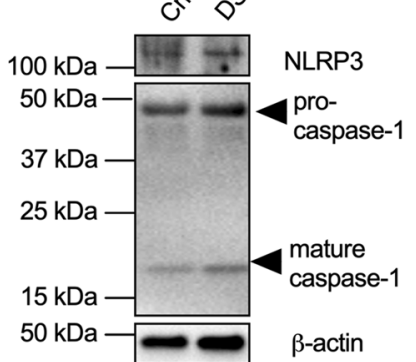

C

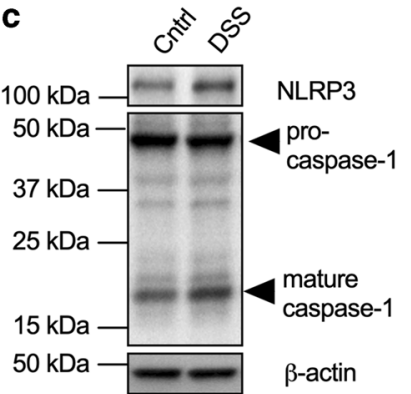

d

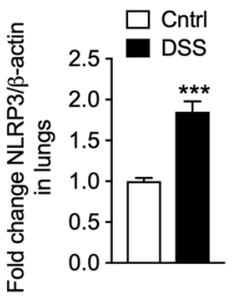

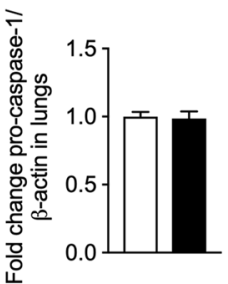

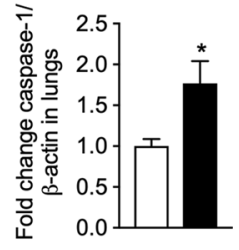

f

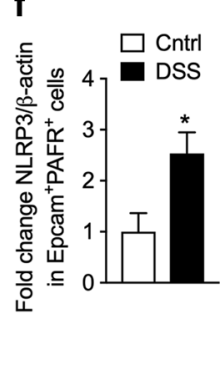

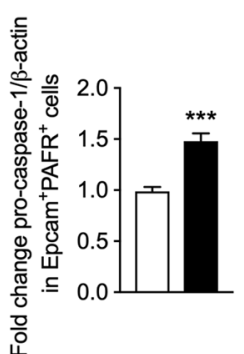

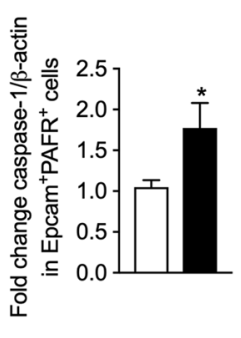

g

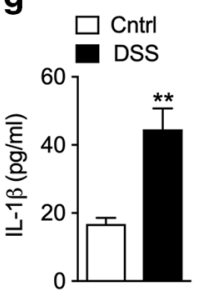

Fig. 3 DSS-induced inflammation and activated inflammasomes in alveolar epithelial cells and mouse whole lungs. Protein levels of TNF (a) and IL-1 $\beta$ (b) proteins in mouse lungs were measured by ELISA. c NLRP3, pro-caspase-1, mature caspase-1, and $\beta$-actin proteins were measured in mouse colon after DSS challenge by immunoblot. $\mathbf{d}$ Fold change of the proteins normalized to $\beta$-actin. CD45-Epcam ${ }^{+}$PAFR ${ }^{+}$were isolated from the lungs of DSS and control animals and sorted by flow cytometry. e NLRP3, pro-caspase-1, mature caspase-1, and $\beta$-actin proteins were measured in cell lysates by immunoblot. $\mathbf{f}$ Fold change of the proteins normalized to $\beta$-actin. $\mathbf{g}$ IL- $1 \beta$ protein in mouse epithelial cells was measured by ELISA. $n=6-8$, mean \pm SEM. Statistical analysis was assessed by Student's $t$-test. ${ }^{*} P<0.05,{ }^{*} P<0.01,{ }^{* * *} P<0.001$ compared to control mice

epithelial cells in COPD patients ${ }^{30}$ and PAFR mRNA levels are increased in asthmatics compared to controls. ${ }^{19}$ The ChoP moiety of bacterial lipoteichoic acid has a strong binding affinity to PAFR and this finding, coupled with observed co-localization of PAFR with Gram-positive bacteria such as Streptococcus pneumonia in pulmonary disease, ${ }^{31}$ has led to the suggestion that microbes may exploit PAFR through molecular mimicry ${ }^{15,16}$ to gain access to the serosa during mucosal inflammation. ${ }^{18}$ However, ChoP expression is regulated by phase variation in both Gram-negative and Grampositive bacteria and that upregulation of ChoP confers survival advantages during colonization. ${ }^{22}$ Thus ChoP may instead be a pathogen-associated molecular pattern recognized by PAFR at the onset of ischemic inflammation to prevent or limit bacterial colonization. Supporting this, ChoP is also recognized by circulating C-reactive protein which leads to activation of the compliment response. ${ }^{32}$ Here, our data show that NLRP3mediated inflammasome activation is prevented both in vivo and in vitro by PAFR antagonism. Fillon et al. have previously proposed PAFR as a PRR that recognizes intravascular ChoPcontaining cell wall components. ${ }^{33}$ This suggests that PAFR induced inflammasome responses may occur during colitisassociated bacteraemia and that colonization of the lung is not critical to the response. This may explain why antibiotic treatment prevented lung NLRP3 inflammasome activation but did not reduce PAFR expression, as cell wall components may be sufficient to increase PAFR, but live-bacteria are required to induce an inflammasome response. Additionally, toll-like receptors (TLR), particularly TLR2 and TLR4, may be important in mediating the PAFR inflammasome response. Studies by Knapp et al. have shown the relative importance of TLR2 over TLR4 and PAFR in in regulating inflammatory responses to lipoteichoic acid (LTA) during pulmonary infection. ${ }^{34}$ In contrast to TLR2, deletion of TLR4 and PAFR only partially protected mice for LTA-induced lung injury. However, TLR2 and TLR4 are basolaterally expressed proteins, while PAFR is apically expressed. ${ }^{18}$ Therefore, PAFR may act as an apical PRR, or alternatively shuttle ChoP-containing bacterial proteins to TLR2 and TLR4 on the basolateral membrane of the epithelium.

It is unclear how the lung is exposed to ChoP positive bacteria during colitis. One possibility is that the loss of intestinal barrier integrity allows bacteria to translocate to other organs, such as lung via blood vessels. Attempts by these bacteria to colonize the lung may utilize $\mathrm{ChoP}^{22}$ and ChoP-positive opportunistic pathogens such as $P$. aeruginosa are abundant in the $\mathrm{Gl}$ tract. $^{35}$ However, because ChoP may be transiently expressed by bacteria, $^{22}$ it is difficult to demonstrate this in vivo. Alternatively, the local lung microbiota may sense and respond to the altered epithelial cell metabolism due to inflammation. Some microbial species sense inflammatory hypoxia through increases in adenosine signaling, upregulating virulence factors to facilitate cell adhesion. ${ }^{36}$ As epithelial PAFR is also hypoxia-regulated, ${ }^{18}$ PAFRmediated inflammasome activation may represent an important innate response to counteract opportunistic colonization. In support of this hypothesis, we found alveolar epithelial cells to be a major source of PAFR in mouse lungs after DSS challenge and PAFR $^{+}$epithelial cells had increased NLRP3 and IL-1 $\beta$ protein levels.

In our previous studies we have shown that neutrophil-driven lung inflammation is a feature of experimental models of colitis in mice. ${ }^{7}$ It reflects the findings that neutrophil-mediated lung diseases are the most common type of pulmonary manifestation of IBD. ${ }^{10}$ Studies in PAFR ${ }^{-/-}$mice suggest that PAFR is important for the recruitment of neutrophils to the lung in models of infection and experimental asthma. ${ }^{37}$ In agreement with these findings, we found that antagonism of PAFR significantly reduced pulmonary neutrophils and NETosis in the lung during colitis. Recent studies show that PAF-signaling activates human neutrophils and induces NETs. ${ }^{38}$ Release of NETs by neutrophils prevents bacterial dissemination, however some studies suggest that rather than killing bacteria, NET-induced tissue damage may actually facilitate bacterial replication. ${ }^{39}$ This may explain why in pulmonary disease, recognition of ChoP by PAFR is not sufficient to clear 
a

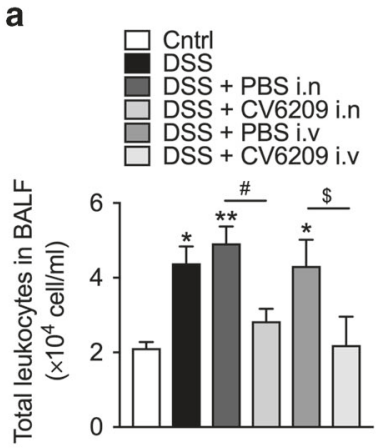

d
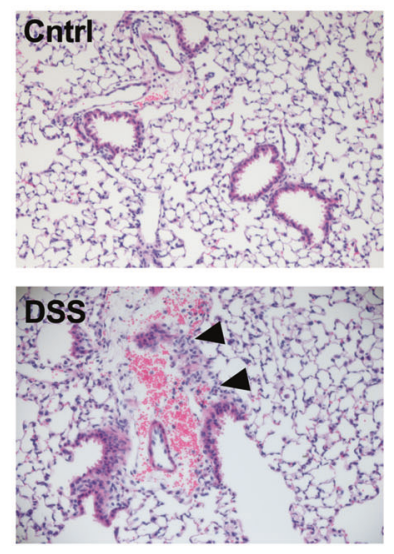

e
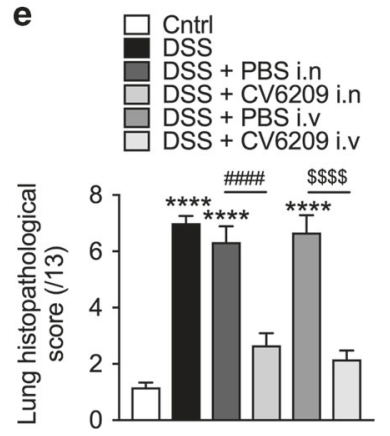

h
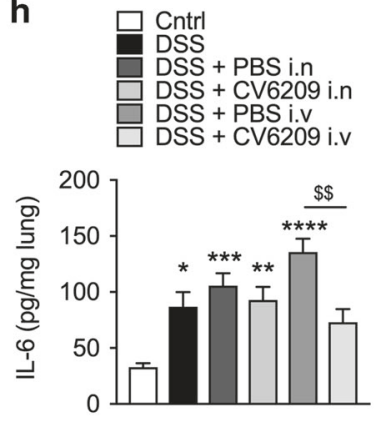

b

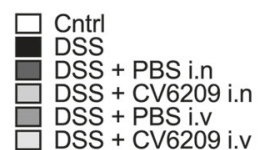

C
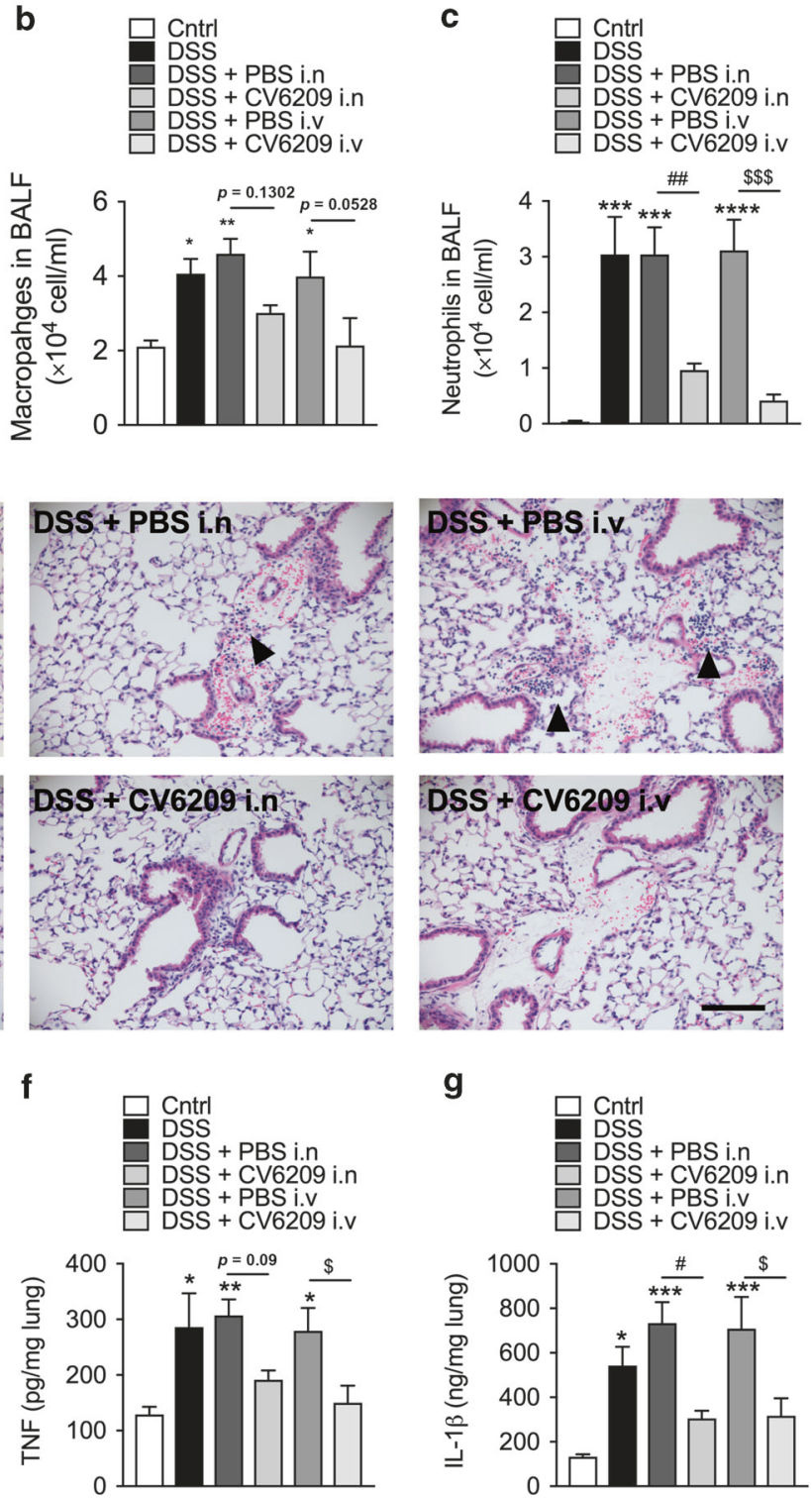

g
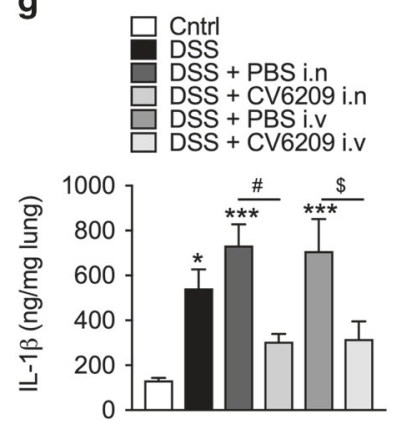

Fig. 4 Influence of PAFR antagonism on colitis-induced lung inflammation in mice. Mice were treated with CV6209 intranasally (i.n) or intravenously (i.v) on day 4, 5, and 6 of DSS model and control mice received i.n. or i.v. PBS. Bronchoalveolar lavage fluid (BALF) was collected, and total leukocytes (a), macrophages (b), and neutrophils (c) were enumerated after cytospin. d Mouse lungs were collected, and lung sections were stained with H\&E. Scale bar: $200 \mu \mathrm{m}$. Arrows indicate inflammation. e Lung inflammatory scores were assessed after 7 days DSS challenge with CV6209 treatments. The levels of TNF (f), IL-1 $\beta(\mathbf{g})$, and IL-6 (h) proteins in mouse lungs were measured by ELISA. $n=6$, mean \pm SEM. Statistical analysis was assessed by one-way ANOVA ${ }^{*} P<0.05,{ }^{* *} P<0.01,{ }^{* * *} P<0.001,{ }^{* * * *} P<0.0001$ compared to control mice. $\# P<0.05$, $\# \# P<0.01$, \#\#\#\#P<0.0001 compared to mice receiving DSS and treated with PBS (i.n). $\$ P<0.05, \$ \$ P<0.001, \$ \$ \$ P<0.0001$ compared to mice receiving DSS and treated with PBS (i.v) 

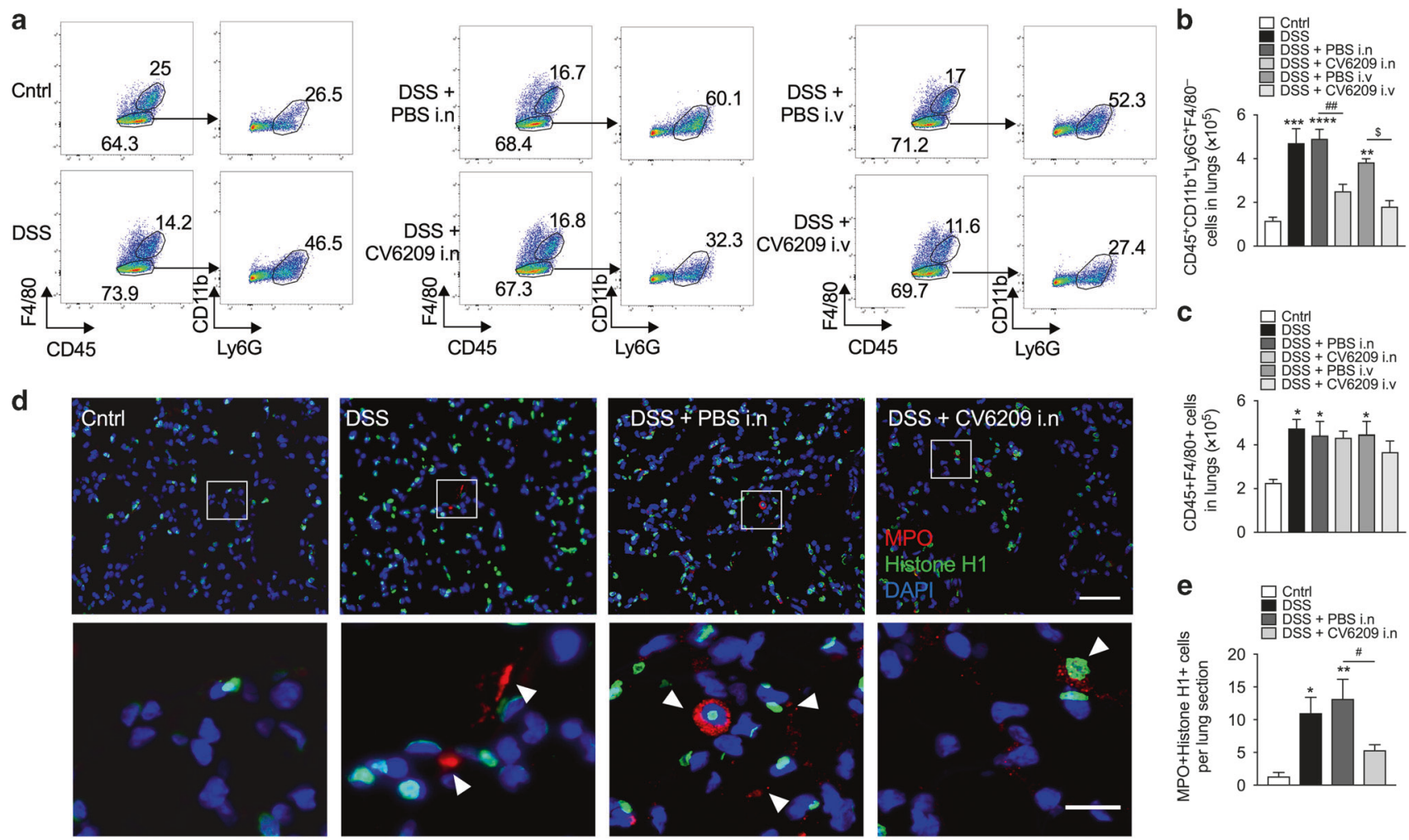

Fig. 5 Influence of PAFR antagonism on neutrophil responses in mouse lungs after DSS challenge. Mice were treated with CV6209 intranasally (i.n) or intravenously (i.v) on day 4,5, and 6 of DSS model and control mice received i.n. or i.v. PBS. Single cell suspensions were obtained from mouse lungs, and stained with CD45, CD11b, F4/80 and Ly6G (a), and CD45 ${ }^{+}$CD $11 b^{+}$Ly6G ${ }^{+} \mathrm{F} 4 / 80^{-}$cells (b) and CD45 ${ }^{+} \mathrm{F} 4 / 80^{+}$cells (c) were enumerated by flow cytometry. $\mathbf{d}$ Mouse lung sections were stained with myeloperoxidase (MPO, red), histone H1 (green) and DAPI (blue) by immunofluorescence. Scale bar: $200 \mu \mathrm{m}$. The indicated regions are shown the expanded at bottom line. Arrows indicate NETs. Scale bar: $20 \mu \mathrm{m}$. e $\mathrm{MPO}^{+}$and histone $\mathrm{H}^{+}$cells were enumerated on mouse lung sections. $n=6$, Results are mean \pm SEM. Statistical analysis was assessed by one-way ANOVA $* P<0.05, * * P<0.01, * * * P<0.001, * * * * P<0.0001$ compared to control mice. \#P<0.05, \#\#P<0.01 compared to mice receiving DSS and treated with PBS (i.n). $\$ P<0.05$ compared to mice receiving DSS and treated with PBS (i.v)

the infection, and in the context of IBD, this may explain why these patients have a higher risk of developing pneumonia. This risk is further increased with use of corticosteroids, and in this respect, it is interesting to note that glucocorticoid use increases PAFR expression in the lungs of patients with COPD. ${ }^{40}$

The activation and release of IL-1 $\beta$ plays a major role in acute inflammatory responses to respiratory infection and promotes clearance of pathogens. However, excessive inflammasome activation and production of IL-1 $\beta$ result in development of several chronic respiratory diseases, including asthma and COPD. ${ }^{41}$ The exact mechanism of NLRP3 inflammasome activation in lung inflammation has remained poorly understood, our finding of a signaling axis between PAFR-NLRP3 and IL-1 $\beta$ mediated neutrophil recruitment is consistent with observations in pulmonary infection models. For instance, $I L-1 \beta^{-/-}$mice fail to recruit neutrophils from bone marrow to lungs in a model of bacteria induced lung infection. ${ }^{42,43}$ With this in mind, therapeutics targeting IL-1 signaling may be of benefit for pulmonary diseases involving PAFR. Our histological analysis in mouse lungs demonstrates that neutrophils are predominantly localize around blood vessel after DSS challenge and that PAFR expression is also localized, but not confined, to lung neutrophils. L-selectin is an important mediator on leukocyte surfaces and is constitutively expressed on the endothelium. ${ }^{44}$ L-selectin plays a role in neutrophil rolling, however neutrophil migration into inflamed tissue is impaired in L-selectin $^{-1-}$ mice. ${ }^{45}$ Because increased PAFR levels are associated with decreased L-selectin expression on neutrophil surfaces PAFR may also trap neutrophils within the lung, further exacerbating inflammation. ${ }^{46}$
Taken together, our data show that PAFR plays critical roles in colitis-induced lung inflammation (Supplementary Fig. S6). Our data is the first to demonstrate that PAFR regulates IL-1 $\beta$ protein activation via the NLRP3 inflammasome signaling pathway. PAFR may act as an inflammasome-activating PRR during colonization of the lung following colitis-induced bacteraemia. Inhibition of PAFR reduced inflammation in mouse lungs after DSS challenge and PAFR may represent a previously unappreciated mediator of secondary lung inflammation in conditions such as IBD and acute lung inflammation.

\section{METHODS}

Experimental colitis-induced lung inflammation

For the DSS colitis model, WT female C57BL/6 mice (6-8 weeks old) received $4 \%$ weight/volume dextran sulfate sodium (DSS; $M$. Wt. 36,000-50,000; MP Biomedicals, Santa Ana, USA) in drinking water ad libitum for 7 days as previously described., ${ }^{7,47}$ Age matched controls received normal drinking water. For PAFR antagonism, mice were administered with $2.5 \mathrm{mg} / \mathrm{kg}$ CV6209 (a PAFR antagonist, Cayman Chemical, Michigan, USA) in sterile PBS either intranasally ${ }^{48}$ or intravenously from day 4 to 6 during DSS challenge, while control mice received equal volume of sterile PBS. For antibiotic treatment, mice received amoxicillin/clavulanate potassium ( $5 \mathrm{mg} / \mathrm{kg}$, Sigma-Aldrich, St. Louis, USA) either intranasally or intragastically from day 0 to day 4 of 7 days DSS challenge, and control mice received equal volume of sterile PBS.

For the anti-CD40 model of colitis, $R A G 1^{-1-}$ female mice on C57BL/6 background (6-8 weeks old) were intraperitoneally 

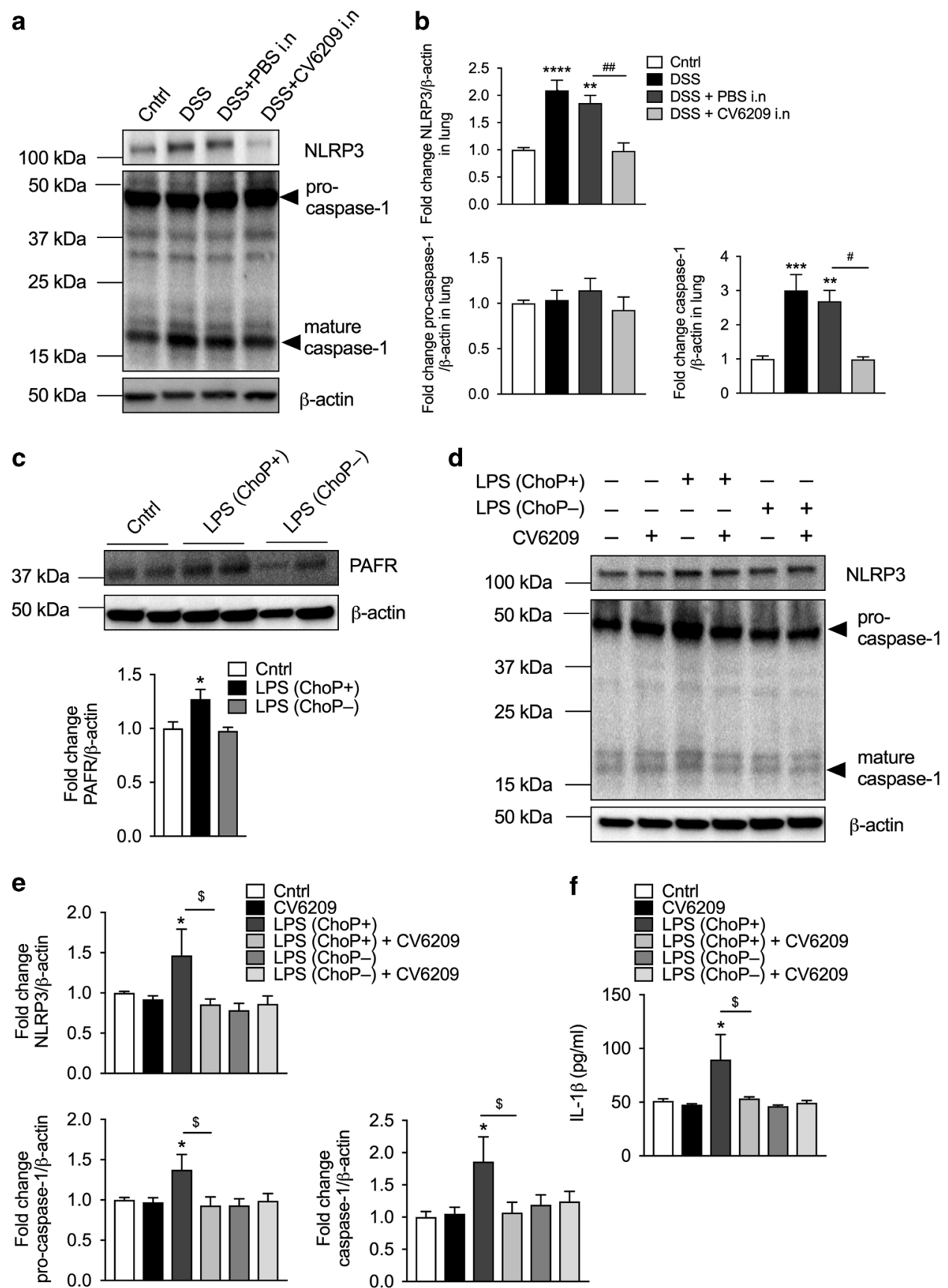

Fig. 6 Activation of NLRP3 inflammasome signaling though PAFR. Mice were treated with CV6209 intranasally (i.n) on day 4, 5, and 6 of DSS model and control mice received i.n. or i.v. PBS. a NLRP3, caspase-1, and $\beta$-actin proteins in whole lungs were assessed by immunoblot, and b fold change of these proteins were determined by densitometry normalized to $\beta$-actin. For in vitro analyses, human alveolar epithelial cells were treated with CV6209 or vehicle and stimulated with phosphorylcholine (ChoP) positive or negative LPS for $4 \mathrm{~h}$. c PAFR proteins in the cell lysates were assessed by immunoblot, and densitometry was normalized to $\beta$-actin. $\mathbf{d}$ NLRP3, caspase- 1 , and $\beta$-actin proteins in the cell lysates were assessed by immunoblot, and e fold change of these proteins was determined by densitometry normalized to $\beta$-actin. $\mathbf{f}$ IL-1 $\beta$ proteins were assessed in cell supernatant by ELISA. $n=5-6$. Results are mean \pm SEM. Statistical analysis was assessed by one-way ANOVA $* P<0.05$, ${ }^{* *} P<0.01,{ }^{* *} P<0.001,{ }^{* * * *} P<0.0001$ as compared to control mice or cell treated with media. $\# P<0.05$, $\# P<0.01$ compared to mice received DSS and treated with PBS (i.n). \$P<0.05 compared to cells received LPS from ChoP positive bacteria

injected with monoclonal anti-CD40 antibody $(200 \mu \mathrm{g} / \mathrm{mouse}$, FGK45, Bxcell, West Lebanon, USA) for 9 days as previously described. ${ }^{49}$ Controls received equal amount of IgG antibody (Bxcell, West Lebanon, USA). All mouse experiments were approved by the Animal Ethics Committee of the University of Newcastle.

\section{Murine endoscopy}

Mucosal inflammation was assessed by colonoscopy on day 3, 5 , and 7 of DSS challenge and on day 2, 4, 6, and 8 of anti-CD40 challenge using the Coloview $^{\circledR}$ small animal gastrointestinal endoscope (Karl Storz Endoscopy, Tuttlingen, Germany). ${ }^{50}$ Representative images were captured from HD videos. 
a

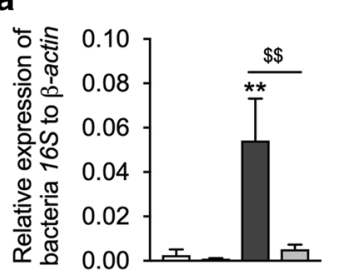

b

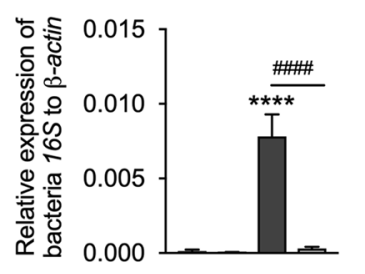

c
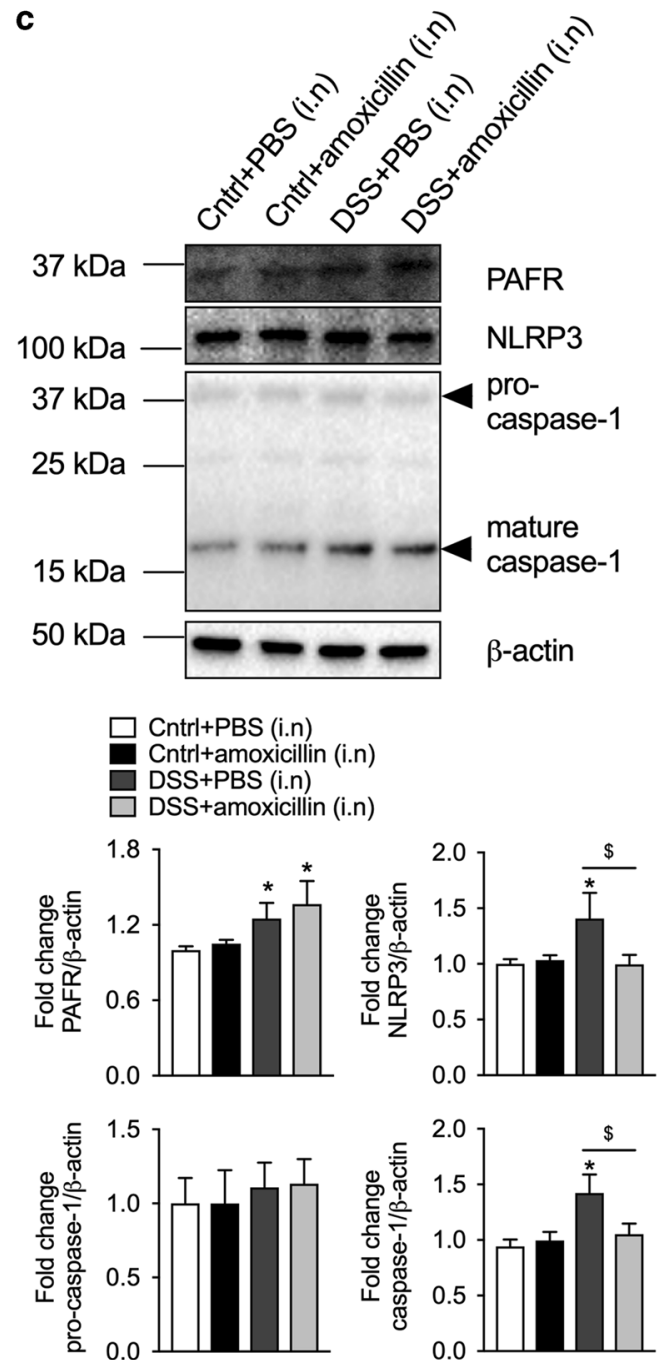
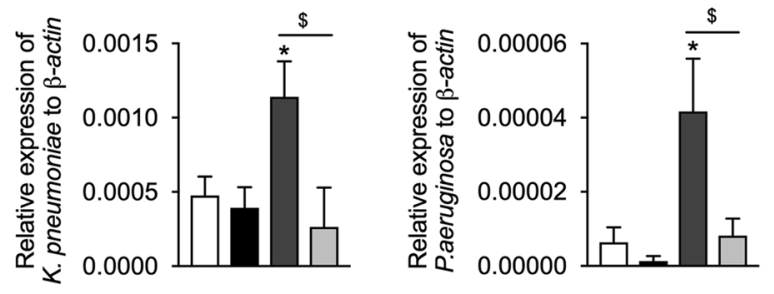

Cntrl+PBS (i.n)

Cntrl + amoxicillin (i.n)

DSS+PBS (i.n)

DSS+amoxicillin (i.n)
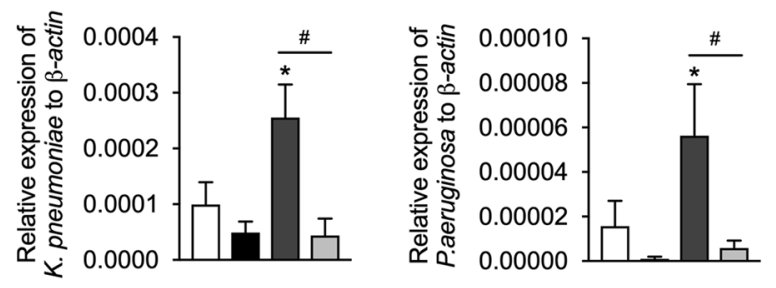

Cntrl+PBS (i.g)

Cntrl + amoxicillin (i.g)

DSS+PBS (i,g)

DSS+amoxicillin (i.g)

d
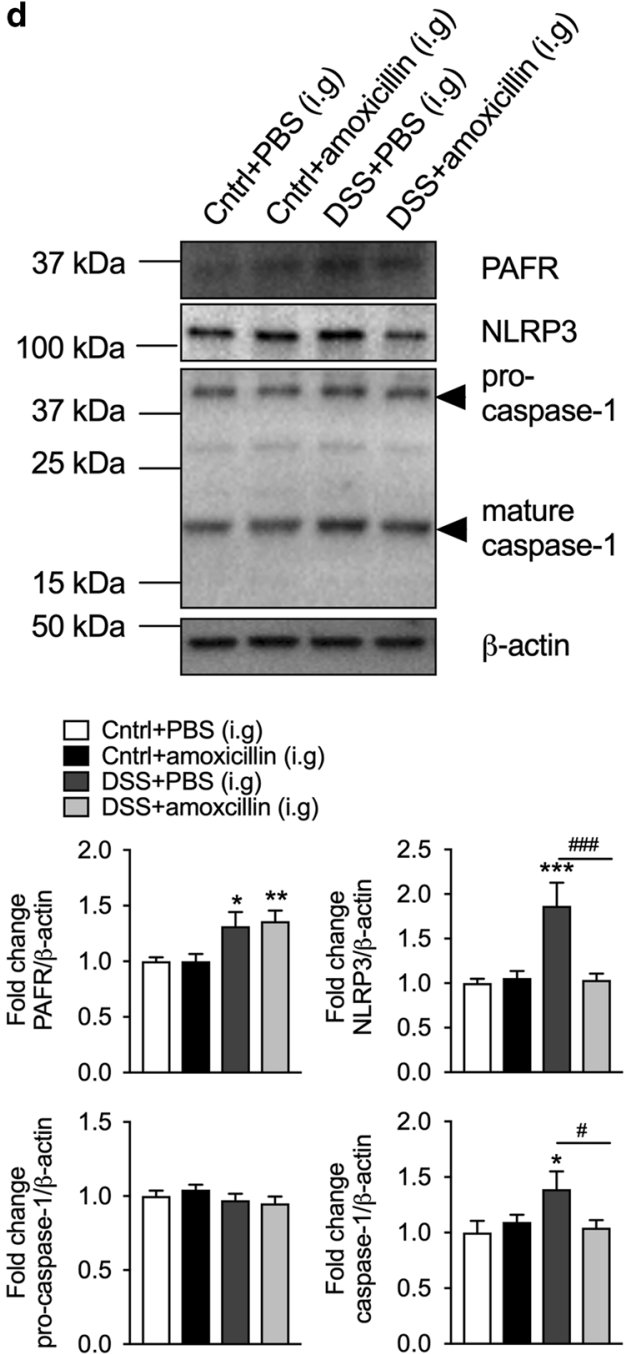

Fig. 7 Influence of antibiotics on bacteria load and the PAFR-NLRP3 inflammasome axis in the lungs of DSS mice. Mice received amoxicillin/ clavulanic acid $(5 \mathrm{mg} / \mathrm{kg}$ ) either intranasally (i.n) or intragastrically (i.g) over the course of DSS challenge. a Total bacteria 16S, $K$. pneumoniae and $P$. aeruginosa expression in mouse lungs after i.n antibiotics. b Total bacteria $165, K$. pneumoniae and $P$. aeruginosa expression in mouse lungs after i.g antibiotics. c PAFR, NLRP3, caspase- 1 and $\beta$-actin proteins in mouse lungs after i.n. antibiotics treatment were assessed by immunoblot (top), and fold change of these proteins were determined by densitometry normalized to $\beta$-actin (bottom). d PAFR, NLRP3, caspase-1, and $\beta$-actin proteins in mouse lungs after i.g. antibiotics treatment were assessed by immunoblot (top), and fold change of these proteins were determined by densitometry normalized to $\beta$-actin (bottom). $n=6$, mean \pm SEM. Statistical analysis was assessed by one-way

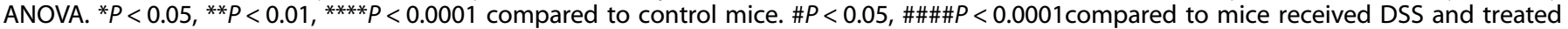
with antibiotics (i.g). $\$ P<0.05, \$ \$ P<0.001$ compared to mice received DSS and treated with antibiotics (i.n) 
Bronchoalveolar lavage fluid (BALF)

BALF were collected by washing mouse airways twice with $500 \mu \mathrm{l}$ PBS, and then incubated with red blood cell lysis buffer $(150 \mathrm{mM}$ $\mathrm{NH}_{4} \mathrm{Cl}, 10 \mathrm{mM} \mathrm{NaHCO}$ and $1.26 \mathrm{mM}$ EDTA) at $4{ }^{\circ} \mathrm{C}$ for $5 \mathrm{~min}$. Total cells in airways were counted after centrifugation $\left(187 \times g, 4^{\circ} \mathrm{C}\right.$, $10 \mathrm{~min})$, and remaining cells were than cyto-centrifugated $(300 \times g$, $5 \mathrm{~min}$ ) on microscope slides. The cells were stained with MayGruwald-Giemsa and identified according to morphological criteria using a light microscope as previously described. ${ }^{48}$

\section{Lung histological scoring}

Mouse tissues were fixed, paraffin-embedded, sectioned (4-6 $\mu \mathrm{m})$, and stained with hematoxylin and eosin (H\&E). Histopathology sections were scored blinded, according to a set of customdesigned criteria as described previously.

\section{mRNA expression}

Mouse lungs were collected, and total RNA were extracted by TRIzol (Invitrogen, Carlsbad, USA) reagent method. RNA (1000 ng) was treated with DNase (Sigma-Aldrich, St. Louis, USA) and reverse-transcribed using iScript according to manufactory's instrument (Biorad, Hercules, USA). Total bacterial 16S (F:CGTCAGCTCGTGTTGTGAAA; R:GGCAGTCTCCTTGAGTTCC), Klebsillia. pneumoniae (F:CATCTCGATCTGCTGGCCAA; R:GCGCGGATCCA GCGATTGGA), Pseudomonas aeruginosa (F:AGTTGTCGCGGCGC TACTAC; R:GCTCACCTGGATCTGGTCC) and mouse $\beta$-actin (F:GG AGAAAATCTGGCACCACA; R:AGAGGCGTACAGGGATAGCA) mRNA expressions were determined using real-time qPCR by a CFX touch real-time PCR detection system (Biorad, Hercules, USA). Relative expressions of target mRNA expressions were determined to the reference $\beta$-actin gene.

\section{Protein extraction}

Proteins were extracted from mouse tissue homogenates and cell culture lysates using radio immunoprecipitation assay buffer (RIPA; Sigma-Aldrich, St. Louis, USA), supplemented with protease and phosphatase inhibitors (Thermo Fisher Scientific, Waltham, USA). Homogenization was performed using a TissueLyser LT (Qiagen, Hiden, Germany) as previously described. ${ }^{51}$ Lysed samples were centrifuged $\left(8000 \times g, 10 \mathrm{~min}, 4^{\circ} \mathrm{C}\right)$ and supernatants were collected from centrifuged for assessment by enzyme linked immunoblot and immunosorbent assay (ELISA). Total protein concentrations were measured using Pierce BCA Protein Assay Kit (Thermo Fisher Scientific, Waltham, USA) according to manufacturer's instructions.

\section{Immunoblot}

Total proteins were separated by electrophoresis using MiniProtean TGX stain free polyacrylamide gels (Bio-Rad, Hercules, USA) and transferred onto polyvinylidene difluoride (PVDF) membranes. The membranes were blocked with 5\% BSA (SigmaAldrich, St. Louis, USA) for $2 \mathrm{~h}$ at room temperature (RT), and then incubated with anti-PAFR (1:1000, sc-8744, Santa Cruz Biotechnology, Santa Cruz, USA), anti-NLRP3 (1:1000, ab4207, Abcam, Cambridge, UK), anti-caspase-1 (1:1000, NBP1-45433, Novus biologicals, Littleton, USA), anti- $\beta$-actin (1:10,000, ab8227, Abcam, Cambridge, UK) and anti-vinculin (1:5000, MAB6896, R\&D System) at $4{ }^{\circ} \mathrm{C}$ overnight. Blots were incubated with anti-goat or antirabbit IgG HPR conjugated antibodies (R\&D System, Minneapolis, USA). Images of immunoblots were captured with a ChemiDoc MP System (Bio-Rad, Hercules, USA). Densitometry analysis was performed relative to houskeeping proteins $\beta$-actin or vinculin, using ImageJ ( $\mathrm{NIH}$, Bethesda, USA) as previously described. ${ }^{51}$

\section{Immunofluorescence}

Longitudinal mouse lung sections were deparaffinized and incubated with citrate buffer $(10 \mathrm{mM}$ citrate acid, $0.05 \%$ tween $20, \mathrm{pH} 6)$ at $100{ }^{\circ} \mathrm{C}$ for antigen retrieval $(35 \mathrm{~min})$. The slides were then blocked with casein (Thermo Fisher, Waltham, USA) at RT for $1 \mathrm{~h}$, and incubated with anti-PAFR (1:50, Santa Cruz Biotechnology, Santa (ruz, USA) at $4{ }^{\circ} \mathrm{C}$ overnight and followed by anti-goat Cy 3 conjugated secondary antibody (R\&D System, Minneapolis, USA) at RT for $1 \mathrm{~h}$. The slides were then incubated with F4/80 (1:100, FITC conjugated) and Ly6G (1:100, BV421 conjugated, BD Biosciences, Franklin Lakes, USA) at RT for $1 \mathrm{~h}$. Some slides were stained with myeloperoxidase (MPO, 1:100, PA5-16672, Thermo Fisher, Waltham, USA) and histone H1 (1:200, Mab3864, Merck, Kenilworth, USA) antibodies and followed by anti-rabbit Cy3 and anti-mouse FITC secondary antibodies from Abcam, Cambridge, UK. Nuclei were stained with DAPI (Thermo Fisher, Waltham, USA). Random pictures (20 images under 40x magnification) were taken from mouse lung sections, and $\mathrm{MPO}^{+}$and histone $\mathrm{H}^{+}$cells were enumerated using ImageJ ( $\mathrm{NIH}$, Bethesda, USA). All fluorescent signals were quantified using an Axio Imager M2 microscope (Zeiss, Oberkochen, German) as previously described. ${ }^{48}$

\section{ELISA}

The concentrations of TNF, IL-1 $\beta$, and IL- 6 in mouse lung and colon homogenates and human cell lysates were determined using DuoSet ELISA kits (R\&D systems, Minneapolis, USA) according to manufacturer's instructions.

\section{Flow cytometry}

Single cell suspensions were obtained from mouse lungs using gentleMACS ${ }^{\text {TM }}$ Dissociators (Mitenyi Biotec, Australia). Cells were incubated with Fc blocker (BD Pharmingen, Franklin Lakes, USA) and stained with CD45 (conjugated with PE-Cy7, BD Biosciences, Franklin Lakes, USA), F4/80 (Conjugated with FITC, BD Pharmingen, Franklin Lakes, USA), CD11b (conjugated with Alexa Fluor 700, BD pharmingen, Franklin Lakes, USA) and Ly6G (conjugated with BV510) antibodies at $4{ }^{\circ} \mathrm{C}$ for $30 \mathrm{~min}$. For some experiments, cells were separated by MojoSort ${ }^{\mathrm{TM}}$ Mouse CD45 Nanobeads (BioLegend, San Diego, USA) according to manufacturer's instrument. All CD45 negative cells were stained with Epcam (conjugated with BV421, BioLegend, San Diego, USA) and PAFR (ab104162, Abcam, Cambridge, UK) at $4{ }^{\circ} \mathrm{C}$ for $30 \mathrm{~min}$, and then followed by anti-rabbit FITC conjugated secondary antibody at $4{ }^{\circ} \mathrm{C}$ for $30 \mathrm{~min}$. CD45 positive cells were stained with $\mathrm{F} 4 / 80$ (BUV395, BD pharmingen, Franklin Lakes, USA), Ly6G (PE-CF594, BioLegend, San Diego, USA), CD11b (BV480, BioLegend, San Diego, USA), CD11C (PE, BioLegend, San Diego, USA), SigF (BV421, BioLegend, San Diego, USA), CD45 (PE-Cy7, BD Biosciences, Franklin Lakes, USA) and PAFR (Abcam, Cambridge, UK) at $4{ }^{\circ} \mathrm{C}$ for $30 \mathrm{~min}$, and then followed by anti-rabbit FITC conjugated secondary antibody at $4{ }^{\circ} \mathrm{C}$ for $30 \mathrm{~min}$. Flow cytometric analysis was performed using a BD LSRFortessa ${ }^{\mathrm{TM}}$ flow cytometer with FACSDiva software (BD Biosciences, Franklin Lakes, USA). Data was analysed using FlowJo software (Tree Star Inc., Ashland, Oregon, USA) as previously described. ${ }^{50} \mathrm{CD} 45^{-} \mathrm{Ep}-$ $\mathrm{cam}^{+} \mathrm{PAFR}^{+}$cells were sorted by a BD FACSAria III cytometer and collected into DMEM medium. Cell lysates were collected, and total proteins were extracted for immunoblot. For cell sorting and flow cytometry assays, all samples were stained and analysed by flow cytometry within $3 \mathrm{~h}$ without fixation and live/ dead cell stain.

\section{Cell culture}

Human alveolar basal epithelial cell line (A549, ATCC, USA) were cultured in DMEM medium contained $10 \%$ fetal bovine serum, $1 \%$ non-essential amino acids, 100 units $/ \mathrm{ml}$ penicillin and $100 \mathrm{mg} / \mathrm{ml}$ streptomycin at $37{ }^{\circ} \mathrm{C}$ with $5 \% \mathrm{CO}_{2}$. Cells were treated with $100 \mu \mathrm{M}$ CV6209 (Cayman Chemical, Ann Arbor, USA) $1 \mathrm{~h}$ before a 4-h LPS challenge. LPS was sourced from $P$. aeruginosa (ChoP positive, $1 \mu \mathrm{g} / \mathrm{ml}$, Sigma-Aldrich, St. Louis, USA) or from E. coli (ChoP negative, $1 \mu \mathrm{g} / \mathrm{ml}$, Sigma-Aldrich, St. Louis, USA). Control groups received equal volume of media. 
Statistical analysis

Results are presented as standard error of the mean (SEM). For mouse studies, 6-8 mice were used per group, sourced from duplicate experiments. Cell culture experiments were repeated at least three times for each time point. Comparisons between two groups were made using unpaired $t$-tests. Comparisons between multiple groups were made using a one-way analysis of variance (ANOVA) with Bonferroni comparisons. For analysis of data with two independent variables such as weight loss data, a two-way ANOVA was utilized. Analyses were performed using the GraphPad Prism Software (San Diego, USA).

\section{ACKNOWLEDGEMENTS}

The authors acknowledge support from the bioresource unit at the University of Newcastle and Hunter Medical Research Institute (HMRI). This work was supported by National Health and Medical Research Council (NHMRC) of Australia, Cancer Institute NSW and a Bowel of the Ball project grant from HMRI.

\section{AUTHOR CONTRIBUTIONS}

S.K. and G.L. participated in the design of the concept, hypothesis and aims of the study and drafting of the manuscript. G.L. performed in vivo experiments. G.L., A.H., J.B., and G.B. performed in vitro and molecular experiments. A.M., B.G., K.M., K.F., and R.N assisted with mouse experiments. H.T. assisted flow cytometry analysis. M.F., and S.M. assisted with data analysis. P.F., P.W., and P.M.H. assisted with concept, experimental design and manuscript editing. All the authors read and approved the final manuscript.

\section{ADDITIONAL INFORMATION}

The online version of this article (https://doi.org/10.1038/s41385-019-0163-3) contains supplementary material, which is available to authorized users.

Competing interests: The authors declare no competing interests.

Publisher's note: Springer Nature remains neutral with regard to jurisdictional claims in published maps and institutional affiliations.

\section{REFERENCES}

1. Ananthakrishnan, A. N. Epidemiology and risk factors for IBD. Nat. Rev. Gastroenterol. Hepatol. 12, 205-217 (2015).

2. Papadakis, K. A. \& Targan, S. R. Role of cytokines in the pathogenesis of inflammatory bowel disease. Annu. Rev. Med. 51, 289-298 (2000).

3. Lodes, M. J. et al. Bacterial flagellin is a dominant antigen in Crohn disease. J. Clin. Invest. 113, 1296-1306 (2004).

4. Pastor Rojo, O. et al. Serum lipopolysaccharide-binding protein in endotoxemic patients with inflammatory bowel disease. Inflamm. Bowel Dis. 13, 269-277 (2007).

5. Gardiner, K. R. et al. Significance of systemic endotoxaemia in inflammatory bowel disease. Gut 36, 897-901 (1995).

6. Keely, S. et al. Contribution of epithelial innate immunity to systemic protection afforded by prolyl hydroxylase inhibition in murine colitis. Mucosal Immunol. 7, 114-123 (2014).

7. Mateer, S. W. et al. IL-6 drives neutrophil-mediated pulmonary inflammation associated with bacteremia in murine models of colitis. Am. J. Pathol. 188, 1625-1639 (2018)

8. Black, H., Mendoza, M. \& Murin, S. Thoracic manifestations of inflammatory bowel disease. Chest 131, 524-532 (2007).

9. Keely, S., Talley, N. J. \& Hansbro, P. M. Pulmonary-intestinal cross-talk in mucosal inflammatory disease. Mucosal Immunol. 5, 7-18 (2012).

10. Mateer, S. W. et al. Potential mechanisms regulating pulmonary pathology in inflammatory bowel disease. J. Leukoc. Biol. 98, 727-737 (2015).

11. Herrlinger, K. R. et al. Alterations in pulmonary function in inflammatory bowel disease are frequent and persist during remission. Am. J. Gastroenterol. 97, 377-381 (2002).

12. Peyrin-Biroulet, L. et al. Efficacy and safety of tumor necrosis factor antagonists in Crohn's disease: meta-analysis of placebo-controlled trials. Clin. Gastroenterol. Hepatol. 6, 644-653 (2008).

13. Liu, Y. et al. Lung and intestine: a specific link in an ulcerative colitis rat model. Gastroenterol. Res. Pract. 2013, 124530 (2013).
14. Aydin, B. et al. Investigation of pulmonary involvement in inflammatory bowel disease in an experimental model of colitis. Korean J. Intern. Med. 31, 853-859 (2016).

15. Sethi, S. \& Murphy, T. F. Infection in the pathogenesis and course of chronic obstructive pulmonary disease. N. Engl. J. Med. 359, 2355-2365 (2008).

16. Kc, R., Shukla, S. D., Walters, E. H. \& O'Toole, R. F. Temporal upregulation of host surface receptors provides a window of opportunity for bacterial adhesion and disease. Microbiology 163, 421-430 (2017).

17. Fernandes, E. S. et al. Cytokines and neutrophils as important mediators of platelet-activating factor-induced kinin B1 receptor expression. Br. J. Pharmacol. 146, 209-216 (2005).

18. Keely, S. et al. Hypoxia-inducible factor-dependent regulation of plateletactivating factor receptor as a route for gram-positive bacterial translocation across epithelia. Mol. Biol. Cell 21, 538-546 (2010).

19. Shirasaki, H. et al. Expression of platelet-activating factor receptor mRNA in human and guinea pig lung. Am. J. Respir. Cell Mol. Biol. 10, 533-537 (1994).

20. Lv, X. X. et al. Cigarette smoke promotes COPD by activating platelet-activating factor receptor and inducing neutrophil autophagic death in mice. Oncotarget $\mathbf{8}$, 74720-74735 (2017)

21. Clark, S. E. \& Weiser, J. N. Microbial modulation of host immunity with the small molecule phosphorylcholine. Infect. Immun. 81, 392-401 (2013).

22. Clark, S. E., Snow, J., Li, J., Zola, T. A. \& Weiser, J. N. Phosphorylcholine allows for evasion of bactericidal antibody by Haemophilus influenzae. PLoS Pathog. 8, e1002521 (2012).

23. O'Toole, R. F., Shukla, S. D. \& Walters, E. H. Does upregulated host cell receptor expression provide a link between bacterial adhesion and chronic respiratory disease? J. Transl. Med. 14, 304 (2016).

24. Hyland, I. K., O'Toole, R. F., Smith, J. A. \& Bissember, A. C. Progress in the development of platelet-activating factor receptor (PAFr) antagonists and applications in the treatment of inflammatory diseases. ChemMedChem 13, 1873-1884 (2018).

25. Mombaerts, P. et al. RAG-1-deficient mice have no mature B and T lymphocytes. Cell 68, 869-877 (1992).

26. Terashita, Z., Imura, Y., Takatani, M., Tsushima, S. \& Nishikawa, K. CV-6209, a highly potent antagonist of platelet activating factor in vitro and in vivo. J. Pharmacol. Exp. Ther. 242, 263-268 (1987).

27. Brinkmann, V. \& Zychlinsky, A. Neutrophil extracellular traps: is immunity the second function of chromatin? J. Cell Biol. 198, 773-783 (2012).

28. Halverson, T. W., Wilton, M., Poon, K. K., Petri, B. \& Lewenza, S. DNA is an antimicrobial component of neutrophil extracellular traps. PLoS Pathog. 11, e1004593 (2015).

29. Cundell, D. R., Gerard, N. P., Gerard, C., Idanpaan-Heikkila, I. \& Tuomanen, E. I. Streptococcus pneumoniae anchor to activated human cells by the receptor for platelet-activating factor. Nature 377, 435-438 (1995).

30. Shukla, S. D., Muller, H. K., Latham, R., Sohal, S. S. \& Walters, E. H. Plateletactivating factor receptor (PAFr) is upregulated in small airways and alveoli of smokers and COPD patients. Respirology 21, 504-510 (2016).

31. Radin, J. N. et al. beta-Arrestin 1 participates in platelet-activating factor receptormediated endocytosis of Streptococcus pneumoniae. Infect. Immun. 73, 7827-7835 (2005)

32. Gao, R. et al. C-reactive protein mediating immunopathological lesions: a potential treatment option for severe Influenza A diseases. EBioMedicine 22, 133-142 (2017).

33. Fillon, S. et al. Platelet-activating factor receptor and innate immunity: uptake of gram-positive bacterial cell wall into host cells and cell-specific pathophysiology. J. Immunol. 177, 6182-6191 (2006).

34. Knapp, S. et al. Lipoteichoic acid-induced lung inflammation depends on TLR2 and the concerted action of TLR4 and the platelet-activating factor receptor. J. Immunol. 180, 3478-3484 (2008).

35. Okuda, J. et al. Translocation of Pseudomonas aeruginosa from the intestinal tract is mediated by the binding of ExoS to an Na,K-ATPase regulator, FXYD3. Infect. Immun. 78, 4511-4522 (2010).

36. Patel, N. J. et al. Recognition of intestinal epithelial HIF-1alpha activation by Pseudomonas aeruginosa. Am. J Physiol. Gastrointest. Liver Physiol. 292, G134-G142 (2007)

37. Patel, P. S. \& Kearney, J. F. CD36 and platelet-activating factor receptor promote house dust mite allergy development. J. Immunol. 199, 1184-1195 (2017)

38. Yost, C. C., Weyrich, A. S. \& Zimmerman, G. A. The platelet activating factor (PAF) signaling cascade in systemic inflammatory responses. Biochimie 92, 692-697 (2010).

39. Narayana Moorthy, A. et al. In vivo and in vitro studies on the roles of neutrophil extracellular traps during secondary pneumococcal pneumonia after primary pulmonary influenza infection. Front. Immunol. 4, 56 (2013).

40. Long, M. D., Martin, C., Sandler, R. S. \& Kappelman, M. D. Increased risk of pneumonia among patients with inflammatory bowel disease. Am. J. Gastroenterol. 108, 240-248 (2013). 
41. Pinkerton, J. W. et al. Inflammasomes in the lung. Mol. Immunol. 86, 44-55 (2017).

42. Miller, L. S. et al. Inflammasome-mediated production of IL-1beta is required for neutrophil recruitment against Staphylococcus aureus in vivo. J. Immunol. 179, 6933-6942 (2007).

43. $\mathrm{Gu}, \mathrm{Y}$. et al. Activation of interferon-gamma inducing factor mediated by interleukin-1 beta converting enzyme. Science 275, 206-209 (1997).

44. Ball, C. J. et al. Hydrogen sulfide reduces neutrophil recruitment in hind-limb ischemia-reperfusion injury in an L-selectin and ADAM-17-dependent manner. Plast. Reconstr. Surg. 131, 487-497 (2013).

45. Zollner, O. et al. L-selectin from human, but not from mouse neutrophils binds directly to E-selectin. J. Cell Biol. 136, 707-716 (1997).

46. Mitchell, M. J., Lin, K. S. \& King, M. R. Fluid shear stress increases neutrophil activation via platelet-activating factor. Biophys. J. 106, 2243-2253 (2014).
47. Kominsky, D. J. et al. An endogenously anti-inflammatory role for methylation in mucosal inflammation identified through metabolite profiling. J. Immunol. 186, 6505-6514 (2011).

48. Liu, G. et al. Airway remodelling and inflammation in asthma are dependent on the extracellular matrix protein fibulin-1c. J. Pathol. 243, 510-523 (2017).

49. Uhlig, H. H. et al. Differential activity of IL-12 and IL-23 in mucosal and systemic innate immune pathology. Immunity 25, 309-318 (2006).

50. Marks, E. et al. Regulation of IL-12p40 by HIF controls Th1/Th17 responses to prevent mucosal inflammation. Mucosal Immunol. 10, 1224-1236 (2017).

51. Liu, G et al. Fibulin-1 regulates the pathogenesis of tissue remodeling in respiratory diseases. JCI Insight 1, e86380 (2016). 